\title{
RECOLLECTION
}

\section{"Light" of the spinach major light-harvester structure}

\author{
Min-Ze Jia (ه) \\ Institute of Biophysics, Chinese Academy of Sciences, Beijing 100101, China \\ $\bowtie$ Correspondence: minzejia@moon.ibp.ac.cn
}

In 2004, Wenrui Chang and his colleagues determined for the first time the atomic structure of the spinach major lightharvesting complex of photosystem II (LHC-II) and published their results in the journal Nature as a full article (Liu et al., 2004). A figure showing the structural model of spinach major LHC-II in a sophisticated and elegant assembly of icosahedral proteoliposome was selected for the cover of that issue of Nature (Fig. 1). Publication of this article immediately drew great attentions from related academe, both domestic and overseas.

Photosynthesis, which is one of the most important and well-known chemical reactions on Earth, requires the involvement of both the light-harvesting system and reaction center to capture and convert solar energy into a chemical form. Light-harvesting antennae collect light quanta and deliver them to the reaction centers where energy conversion takes place. In full sunlight, however, most absorbed energy is not needed and the excess energy can potentially cause photo-damage of the photosynthetic membrane. Therefore, it is vitally essential for plant survival that the antennae are able to switch to specific states where excess energy is safely dissipated as heat. The plant light-harvesting complex of photosystem II (LHC-II) is arguably the most abundant membrane protein on Earth. The LHC-II is the main solar energy collector of plants, but it may also be involved in photoprotection through the control of emission or transfer of incoming light quanta.

In 1994, Kühlbrandt et al. reported the structure of pea LHC-II at 3.4-Å resolution, which was determined by electron crystallography of two-dimensional crystals and provided insightful but limited structural information of LHC-II (Kühlbrandt et al., 1994). Since then, the light-harvesting research community has been eagerly expecting a more detailed and complete 3-D structure of plant light-harvesting complex in order to improve the quantitative studies on the pathways of light energy absorption and transmission, as well as the mechanism of light harvesting regulation to a higher level.
Soon after, the competition in the structural study on plant light-harvester became very intense as many world-famous laboratories in the field were involved in this worldwidely acknowledged difficult project.

In the 1990s, a group of Chinese scientists, led by Chang from the Institute of Biophysics, Chinese Academy of Sciences, joined the competition of X-ray crystallographic study on the plant light-harvesting complex. Their past successful experiences on structural determination of the water-soluble algae light-harvesting protein-pigment complexes laid down a solid technical foundation for taking on this particularly challenging task. Initially, the Chang lab and their collaborators from the Institute of Botany, Chinese Academy of Sciences, overcame the first barrier in the purification of spinach major LHC-II from the thylakoid membrane through differential centrifugation fractionation and size-exclusion chromatography. They obtained monodisperse and stable plant LHC-II preparation suitable for crystallization experiments. Later, they managed to obtain well-ordered crystals of this highly hydrophobic lipoprotein complex through extensive screening and iterative optimization of countless conditions for sample preparation and crystal growth. Then, it came the phasing problem that they tackled by preparing mercuryderivatized crystal and exploited the ten-fold non-crystallographic symmetry for phase refinement and extension. Although often forced to face failure, they never thought about quitting but kept trying to seek the "light" from the spinach major LHC-II. Through six years of painstaking efforts, the Chang lab eventually determined a $2.72-\AA$ resolution structure of the spinach major LHC-II, thus provided a complete atomic view of this intricate complex composed of protein, pigment and lipid molecules.

This work on the atomic structure of spinach major LHC-II revealed for the first time a type III packing pattern of membrane protein crystals, formed by numerous vesicular proteoliposomes with high-order internal symmetry. The nearly complete and highly accurate structural model of 


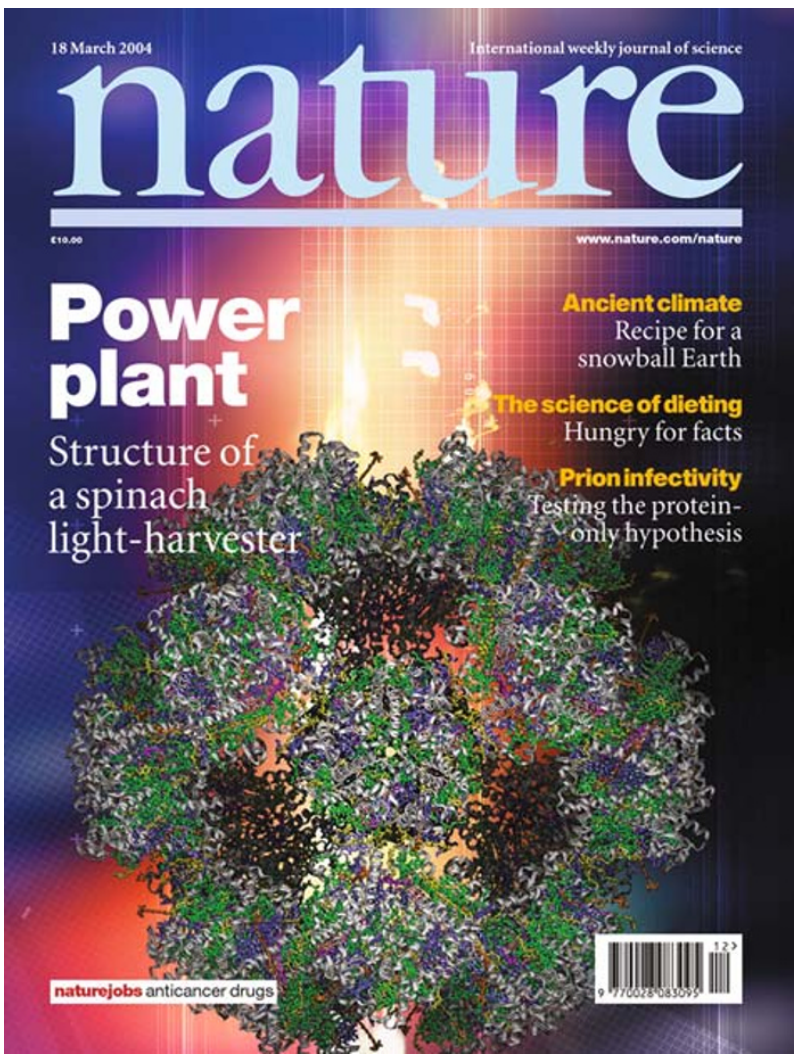

Figure 1. Adapted by permission from Macmillan Publishers Ltd: Nature, March 18, 2004.

LHC-II provided vital structural information for the quantitative study of the energy absorption and transmission pathways in LHC-II. Furthermore, Chang and his colleagues mapped the energy-transferring networks in LHC-II monomer, as well as in and between LHC-Il trimer, based on the spinach major LHCII structure. Finally, a structure-based non-photochemical quenching model was proposed to account for the potential energy quenching sites in LHC-II. Noticeably, it was found in the following year that the spinach LHC-Il crystals were in a dissipative state that exhibited dramatic red shift of fluorescent emission and shorter fluorescence lifetime as compared to the trimeric forms (Pascal et al., 2005). To summarize, the determination of spinach major LHC-II structure indicated that the basic research in China has advanced into the world's advanced level in the fields of structural biology and photosynthesis research.

"After a short excitement, the ordinary and repetitive work continues, day by day," Chang calmly said later. These words represent the truth that it is not achievements themselves that count, but it is the fact that you worked hard towards them. Development in the research field always comes with the steady accumulation of useful knowledge and experiences from the past. It is well-known that in the early 1970 s, a group of Chinese scientists successfully determined the crystal structure of porcine insulin at resolutions of $4-\AA$,
2.5- $\AA$ and 1.8- $\AA$ (Hodgkin, 1975), representing one of the pioneering crystallographic works in the world at that time (Chang participated in the project as one of the principal members). Thirty years later, new outstanding achievements began to emerge in multitudes, including the structures of spinach major LHC-II and the mitochondrial respiratory complex II (Sun et al., 2005) et al., which set the new landmarks for the rapid development of structural biology in China.

\section{REFERENCE}

Hodgkin, D. (1975). Chinese work on insulin. Nature 255, 103.

Kühlbrandt, W., Wang, D.N., Fujiyoshi, Y. (1994). Atomic model of plant light-harvesting complex by electron crystallography. Nature 367, 614-621.

Liu, Z., Yan, H., Wang, K., Kuang, T., Zhang, J., Gui, L., An, X., and Chang, W. (2004). Crystal structure of spinach major lightharvesting complex at 2.72-Å resolution. Nature 428, 287-292.

Pascal, A.A., Liu, Z., Broess, K., Oort, B.V., Amerongen, H.V., Wang, C., Horton, P., Robert, B., Chang W., and Ruban, A. (2005). Molecular basis of photoprotection and control of photosynthetic light-harvesting. Nature 436, 134-137.

Sun, F., Huo, X., Zhai, Y., Wang, A., Xu, J., Su, D., Bartlam, M., and Rao, Z. (2005). Crystal structure of mitochondrial respiratory membrane protein complex II. Cell 121, 1043-1057. 